\title{
Supraceliac Aortic Clamping for Repair of Ruptured Abdominal Aortic Aneurysm in Patients with Short Aortic Neck Length
}

\author{
Koji Furukawa1, Mitsuhiro Yano, Eisaku Nakamura², Masakazu Matsuyama", \\ Shuhei Sakaguchi1, Katsuya Kawagoe', Kunihide Nakamura² \\ ${ }^{1}$ Department of Cardiovascular Surgery, Miyazaki Medical Association Hospital, Miyazaki, Japan \\ ${ }^{2}$ Department of Cardiovascular Surgery, University of Miyazaki, Miyazaki, Japan \\ Email: koji_furukawa@med.miyazaki-u.ac.jp
}

Received 3 December 2015; accepted 8 January 2016; published 11 January 2016

Copyright (C) 2016 by authors and Scientific Research Publishing Inc.

This work is licensed under the Creative Commons Attribution-NonCommercial International License (CC BY-NC).

http://creativecommons.org/licenses/by-nc/4.0/

(c) (i) (9) Open Access

\begin{abstract}
Objective: Prompt bleeding control with proximal aortic clamping and subsequent aortic repair are very important for ruptured abdominal aortic aneurysm. However, unsuitable anatomy, such as short aortic neck length, not only disturbs the means to an expeditious repair, but may also increase morbidity and mortality. In the present study, we aimed to evaluate the efficacy of supraceliac aortic clamping for improving surgical outcomes for patients with ruptured abdominal aortic aneurysm, who have a short aortic neck length. Method: Between April 2010 and September 2015, eighteen patients underwent emergent open surgical repair of ruptured abdominal aortic aneurysm. Eight patients with a short aortic neck length underwent supraceliac aortic clamping, and 10 underwent infrarenal aortic clamping. Results: The mean supraceliac aortic clamping time was 30 \pm 7 minutes. There was 1 operative death in the infrarenal aortic clamping group due to respiratory failure, and the overall operative mortality was $6 \%$. There were no significant differences between the 2 groups with respect to postoperative complication rates or mortality. Furthermore, there were no significant differences in variables of renal function between the 2 groups, throughout the study period. Conclusion: Supraceliac aortic clamping was associated with minimal mortality and morbidity, but not with harmful effects on postoperative renal function. Thus, supraceliac aortic clamping can be safely applied for ruptured abdominal aortic aneurysm with short aortic neck length.
\end{abstract}

\section{Keywords}

Ruptured Abdominal Aortic Aneurysm, Short Aortic Neck Length, Open Surgical Repair, Supraceliac Aortic Clamping

How to cite this paper: Furukawa, K., Yano, M., Nakamura, E., Matsuyama, M., Sakaguchi, S., Kawagoe, K. and Nakamura, K. (2016) Supraceliac Aortic Clamping for Repair of Ruptured Abdominal Aortic Aneurysm in Patients with Short Aortic Neck Length. World Journal of Cardiovascular Surgery, 6, 5-13. http://dx.doi.org/10.4236/wjcs.2016.61002 


\section{Introduction}

As the mean age of our population continues to rise, the incidence of abdominal aortic aneurysm (AAA) is expected to increase concomitantly. For small aneurysms, the median rate of increase in aneurysm diameter is 0.32 $\mathrm{cm}$ per annum. When the diameter expands to more than $5-6 \mathrm{~cm}$, AAAs will rupture at the rate of $5 \%$ to $7 \%$ per annum; the five-year rupture rate for aneurysms $7 \mathrm{~cm}$ or larger is upwards of 95\%. Rupture remains the most morbid and often fatal complication of AAA; thus, the early identification and efficacious management of this pathology continues to be of vital importance [1]. However, the mortality rate of open surgical repair of ruptured AAA (rAAA) remains remarkably high (range, 23\% - 54\%) [2]-[5], which is in contrast with the relatively good outcomes obtained for the repair of non-ruptured AAAs [5] [6]. Recently, endovascular aneurysm repair (EVAR) has been shown to be a promising alternative to open surgical repair for not only non-ruptured AAA [7] but also rAAA [2]-[4]. However, unsuitable anatomy, such as short aortic neck length, precludes EVAR, and open surgical repair is the preferred procedure for such cases. Nevertheless, there are some potential risks associated with dissection, aortic clamping, and anastomosis around short aortic necks, such as arterial embolism, second rupture, and anastomotic bleeding. We have performed supraceliac (SC) aortic clamping for the expeditious and safe repair of rAAA in patients with a short aortic neck length. In the present study, we aimed to evaluate our surgical results for rAAA, and in particular, to determine the effect of SC aortic clamping on renal function, morbidity, and outcomes compared to the effects and outcomes for infrarenal (IR) aortic clamping.

\section{Patients and Methods}

Between April 2010 and September 2015, 18 patients underwent emergent surgical repair of rAAA, including 2 cases of para-anastomotic aneurysm rupture, at Miyazaki Medical Association Hospital. Written informed consent was obtained for each patient, and the study design was approved by the Miyazaki Medical Association Hospital review board. All patients underwent open repair because our hospital did not perform EVAR for AAA until March 2015. All patients underwent preoperative computed tomography (CT), and rupture was defined as the visualization of extraluminal blood by CT. Our policy is to perform SC aortic clamping for cases of rAAA, in which patients have a short distance between the renal artery and the aneurysm (short aortic neck length), and for whom it can be difficult to achieve a precise repair with IR cross-clamping. Consequently, 8 patients (44\%) underwent SC aortic clamping (SC group) and 10 (56\%) underwent IR aortic clamping (IR group). Figure 1 shows a CT image of an rAAA with short aortic neck length that was evaluated in the present study.

The preoperative hemodynamic status of patients was defined according to the Rutherford classification, as follows: level 1, no hypotension; level 2, transient hypotension, with a satisfactory response to resuscitation; level 3, incomplete response to resuscitation, with persistent or recurrent hypotension and/or no restoration of

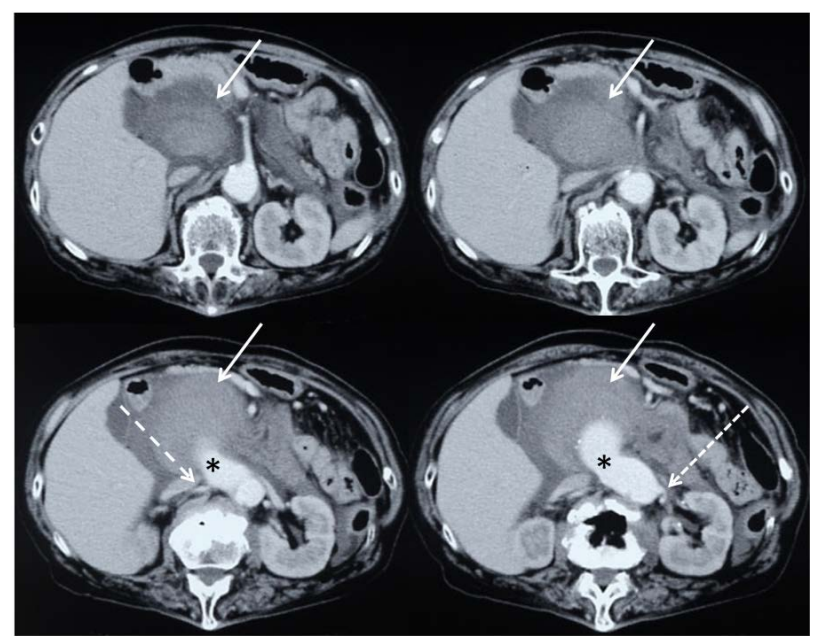

Figure 1. Computed tomography showing abdominal aortic aneurysm with visualization of extraluminal blood (white arrow) and short distance (asterisk) between the renal artery (dashed arrow) and the aneurysm. 
urine output; and level 4, negligible response to resuscitation [8]. The rupture type was defined according to the Fitzgerald classification, as follows: type 1 included intramural bleeding or a small hematoma; type 2 included a hematoma below the renal arteries that included the pelvis; type 3 comprised a hematoma extending above the renal arteries and into the pelvis; and type 4 comprised free blood in the peritoneal cavity detected either by CT or during the operation, or both [9].

All surgery was performed via the transabdominal approach with the patient under general anesthesia. IR aortic clamping was performed according to standard procedures. For SC aortic clamping, the following steps were performed: Initially, the lesser sac was opened to allow opening of the diaphragmatic crura. The crura were split with electrocautery before blunt dissection of the aorta, which allows rapid and accurate clamp placement in the SC aorta. After completing the proximal anastomosis, the graft and proximal aorta were flushed by temporary removal of the aortic clamp at the SC aorta. The graft was then cross-clamped below the anastomosis, and the aortic clamp at the SC aorta was removed to restore circulation into the visceral vessels while the distal anastomosis was completed [10]. All aneurysms, excluding that of one patient with para-anastomotic aneurysm rupture who required left renal artery revascularization, were repaired with proximal anastomosis just below the renal arteries. To prevent abdominal compartment syndrome (ACS), prophylactic temporary abdominal closure (TAC) was performed by sewing a vinyl sheet to the skin to expand the abdominal compartment at the initial operation when it was difficult to achieve primary closure of the abdominal wall or when ACS was most likely to develop upon closure. Inferior mesenteric artery reconstruction was not performed in any of the cases.

Operative mortality was defined as death occurring within 30 days of surgery, regardless of whether the patient had been discharged from the hospital. All patients were followed up until September 2015.

The data are expressed as mean \pm standard deviation. Statistical analyses were performed by using the t-test for continuous variables or with the $\chi^{2}$ test for categorical variables. A probability value of $<0.05$ was considered significant. Cumulative survival was calculated by the Kaplan-Meier method with the dates of surgery and most recent followup.

\section{Results}

The preoperative characteristics of the patients are presented in Table 1 . The mean age of patients was $76 \pm 9$ years (range, 63 - 91 years), and 5 patents were aged $\geq 80$ years. Nine patients (50\%) had a Rutherford classification level of 3 or 4, including 2 patients with circulatory collapse who required emergency tracheal intubation. The mean size of rAAAs was $74 \pm 12 \mathrm{~mm}$ (range, 57 - $100 \mathrm{~mm}$ ), and the mean IR neck length was $18 \pm 9 \mathrm{~mm}$ (range, $0-34 \mathrm{~mm}$ ). Fourteen patients (78\%) had a Fitzgerald classification of type 3 or 4 . There were no significant differences in age, ratio of elderly patients, gender, preoperative hemodynamic status, hematocrit level, or rAAA size between the 2 groups. However, the SC group had a significantly shorter IR neck length than that of the IR group ( $11 \pm 6 \mathrm{~mm}$ vs. $24 \pm 6 \mathrm{~mm}$; $\mathrm{P}<0.01)$. In addition, the SC group was characterized by patients with a rupture of Fitzgerald classification type 3 or 4 , and included 2 cases of para-anastomotic aneurysm rupture.

Table 1. Preoperative characteristics.

\begin{tabular}{ccccc}
\hline & $\begin{array}{c}\text { All patients } \\
(\mathbf{n = 1 8})\end{array}$ & $\begin{array}{c}\text { SC } \text { group } \\
(\mathbf{n = 8}\end{array}$ & $\begin{array}{c}\text { IR group } \\
\text { (n= 10) }\end{array}$ & p-value \\
Age (years) & $76 \pm 9$ & $74 \pm 6$ & $77 \pm 12$ & 0.5 \\
$\geq 80$ years & 5 & 1 & 4 & 0.2 \\
Male (n) & 12 & 4 & 8 & 0.2 \\
Rutherford level 3 or 4 (n) & 9 & 4 & 5 & 0.9 \\
Hct (\%) & $31 \pm 7$ & $31 \pm 10$ & $31 \pm 4$ & 0.7 \\
rAAA size (mm) & $74 \pm 12$ & $72 \pm 11$ & $24 \pm 6$ & $<0.01$ \\
IR neck length (mm) & $18 \pm 9$ & $11 \pm 6$ & 6 & 0.04 \\
\hline
\end{tabular}

SC: supraceliac; IR: infrarenal; Hct: hematocrit level; rAAA: ruptured abdominal aortic aneurysm. 
The operative data are presented in Table 2. The mean dissection time to clamping, which was calculated from skin incision to aortic cross-clamping, was $42 \pm 34$ minutes (range, 17 - 155 minutes). The mean SC aortic clamping time was $30 \pm 7$ minutes (range, 18 - 40 minutes). The mean surgical time was $228 \pm 75$ minutes (range, 138 - 436 minutes). There were no significant differences between the 2 groups with respect to the dissection time and surgical time. In addition, there were neither significant differences in reconstruction style (tube graft replacement or not) nor the amount of intraoperative red blood cell and fresh-frozen plasma transfusion between the 2 groups. Two patients in the IR group underwent TAC during the initial laparotomy because the edema precluded primary closure and there was a high likelihood of the development of ACS upon closure; these patients achieved definitive fascial closure after 2 days.

The postoperative data are presented in Table 3. One 91-year-old patient in the IR group died of respiratory failure on postoperative day 24; thus, the overall operative mortality was 6\%. Paralytic ileus occurred in 6 patients, prolonged mechanical ventilation $\geq 7$ days was required for 3 patients, and re-exploration for bleeding was performed in one patient. Ischemic colitis occurred in one patient who underwent TAC, one month after the surgery; this case was treated conservatively and a successful outcome was achieved. There were no significant differences between the 2 groups with respect to pulmonary and gastrointestinal complications. Moreover, no

Table 2. Operative data.

\begin{tabular}{ccccc}
\hline & $\begin{array}{c}\text { All patients } \\
(\mathbf{n}=\mathbf{1 8})\end{array}$ & $\begin{array}{c}\text { SC group } \\
(\mathbf{n}=\mathbf{8})\end{array}$ & $\begin{array}{c}\text { IR group } \\
(\mathbf{n}=\mathbf{1 0})\end{array}$ & p-value \\
\hline Dissection time to clamping (min) & $42 \pm 34$ & $44 \pm 45$ & $40 \pm 25$ & - \\
SC aortic clamping time (min) & - & $30 \pm 7$ & - & 0.8 \\
Surgical time (min) & $228 \pm 75$ & $228 \pm 93$ & $228 \pm 62$ & 0.9 \\
Tube graft replacement (n) & 8 & 3 & $10 \pm 4$ & 0.8 \\
Intraoperative red blood cell transfusion (U) & $10 \pm 5$ & $9 \pm 7$ & $11 \pm 4$ & 0.3 \\
Intraoperative fresh frozen plasma transfusion (U) & $10 \pm 4$ & $9 \pm 3$ & 2 & 0.2 \\
\hline TAC (n) & 2 & 0 & 5 \\
\hline
\end{tabular}

SC: supraceliac; IR: infrarenal; TAC: temporary abdominal closure.

Table 3. Postoperative data.

\begin{tabular}{|c|c|c|c|c|}
\hline & $\begin{array}{l}\text { All patients } \\
\quad(\mathbf{n}=18)\end{array}$ & $\begin{array}{c}\text { SC group } \\
(\mathbf{n}=\mathbf{8})\end{array}$ & $\begin{array}{l}\text { IR group } \\
(\mathbf{n}=10)\end{array}$ & p-value \\
\hline Operative mortality (n, \%) & $1(6)$ & 0 & $1(10)$ & 0.4 \\
\hline \multicolumn{5}{|l|}{ Complications } \\
\hline Paralytic ileus (n) & 6 & 3 & 3 & 0.7 \\
\hline Mechanical ventilation $\geq 7$ days (n) & 3 & 1 & 2 & 0.7 \\
\hline Re-exploration for bleeding (n) & 1 & 0 & 1 & 0.4 \\
\hline Ischemic colitis (n) & 1 & 0 & 1 & 0.4 \\
\hline $\operatorname{ACS}(n)$ & 0 & 0 & 0 & - \\
\hline Renal replacement therapy (n) & 0 & 0 & 0 & - \\
\hline Hospital stay (day) & $27 \pm 15^{\dagger}$ & $24 \pm 15$ & $29 \pm 16^{\dagger}$ & 0.5 \\
\hline Late death from any cause (n) & 4 & 1 & 3 & 0.3 \\
\hline Long term mortality from rAAA (n) & 0 & 1 & 0 & 0.2 \\
\hline
\end{tabular}

${ }^{\dagger}$ Excludes one patient who died prior to discharge. SC: supraceliac; IR: infrarenal; ACS: abdominal compartment syndrome; rAAA: ruptured abdominal aortic aneurysm. 
ACS occurred and no patient required renal replacement therapy in either group. There was no significant difference in postoperative hospitalization duration between the 2 groups. One patient who underwent graft replacement with left renal artery revascularization due to para-anastomotic aneurysm rupture died of re-anastomotic rupture and 3 patients died of other diseases: thoracic aortic aneurysm, lung cancer, and myocardial infarction. There were no cases of renal failure requiring renal replacement therapy during followup.

The changes in renal function variables are presented in Table 4. The preoperative mean serum blood urea nitrogen (BUN) level was $26 \pm 8 \mathrm{mg} / \mathrm{dL}$ (range, 14 - $50 \mathrm{mg} / \mathrm{dL}$ ), serum creatinine (CR) level was $1.5 \pm 0.7 \mathrm{mg} / \mathrm{dL}$ (range, 0.7 - $3.3 \mathrm{mg} / \mathrm{dL}$ ), and estimated glomerular filtration rate (eGFR) was $38 \pm 13 \mathrm{~mL} / \mathrm{min} / 1.73 \mathrm{~m}^{2}$ (range, 14 - $59 \mathrm{~mL} / \mathrm{min} / 1.73 \mathrm{~m}^{2}$ ); there were no significant differences in preoperative renal function variables between the 2 groups. At discharge, the renal function variables did not deteriorate, and the mean BUN, CR, and eGFR were $26 \pm 15 \mathrm{mg} / \mathrm{dL}, 1.1 \pm 0.4 \mathrm{mg} / \mathrm{dL}$, and $49 \pm 17 \mathrm{~mL} / \mathrm{min} / 1.73 \mathrm{~m}^{2}$, respectively; no significant differences in renal function variables were noted at discharge between the 2 groups.

Figure 2 shows the survival curves in the SC and IR groups. The overall survival rates at 1 and 3 years were $82 \%$ and $62 \%$, respectively; there were no significant differences in the survival rates between the 2 groups.

Table 4. Comparison of blood urea nitrogen, creatinine, and estimated glomerular filtration rate values.

\begin{tabular}{|c|c|c|c|c|}
\hline & $\begin{array}{l}\text { All Patients } \\
\quad(\mathrm{n}=18)\end{array}$ & $\begin{array}{l}\text { SC group } \\
(\mathbf{n}=\mathbf{8})\end{array}$ & $\begin{array}{l}\text { IR group } \\
(\mathbf{n}=10)\end{array}$ & p-value \\
\hline \multicolumn{5}{|l|}{ Preoperative } \\
\hline BUN (mg/dL) & $26 \pm 8$ & $26 \pm 11$ & $25 \pm 6$ & 0.7 \\
\hline CR (mg/dL) & $1.5 \pm 0.7$ & $1.4 \pm 0.6$ & $1.6 \pm 0.7$ & 0.7 \\
\hline eGFR $\left(\mathrm{mL} / \mathrm{min} / 1.73 \mathrm{~m}^{2}\right)$ & $38 \pm 13$ & $38 \pm 11$ & $38 \pm 14$ & 0.9 \\
\hline \multicolumn{5}{|l|}{ At discharge } \\
\hline BUN (mg/dL) & $26 \pm 15^{\dagger}$ & $23 \pm 7$ & $29 \pm 19^{\dagger}$ & 0.4 \\
\hline $\mathrm{CR}(\mathrm{mg} / \mathrm{dL})$ & $1.1 \pm 0.4^{\dagger}$ & $1.1 \pm 0.5$ & $1.2 \pm 0.3^{\dagger}$ & 0.9 \\
\hline eGFR $\left(\mathrm{mL} / \mathrm{min} / 1.73 \mathrm{~m}^{2}\right)$ & $49 \pm 17^{\dagger}$ & $50 \pm 22$ & $48 \pm 13^{\dagger}$ & 0.8 \\
\hline
\end{tabular}

${ }^{\dagger}$ Excludes 1 patient who died prior to discharge. SC: supraceliac; IR: infrarenal; BUN: blood urea nitrogen; CR: creatinine; eGFR: estimated glomerular filtration rate.

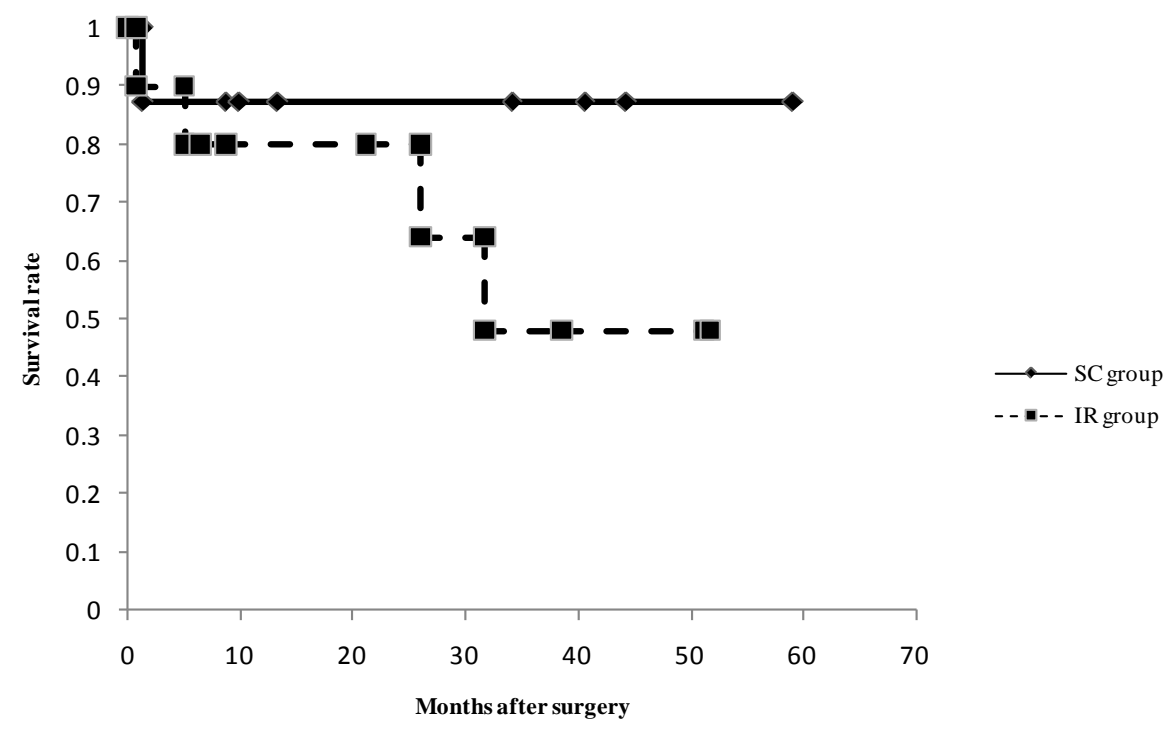

Figure 2. Kaplan-Meier survival curves in the supraceliac (SC [solid line]) and infrarenal (IR [dashed line]) groups. 


\section{Discussion}

Recently, EVAR has shown promise as an alternative to open surgical repair for rAAA, a procedure for which the mortality rate remains high. However, EVAR may not be suitable for all patients because of unsuitable anatomy, such as short aortic length, and open surgical repair is the preferred procedure for such cases. Here, we compared the methods of SC and IR aortic clamping by evaluating the effects and outcomes for each technique among patients with rAAA. The SC group had a significantly shorter IR neck length than that of the IR group. We found no differences in the mean dissection time, surgical time, transfusion requirements, postoperative complications, or mortality between the SC and IR groups. Furthermore, we found that, compared to IR aortic clamping, SC aortic clamping had no harmful effects on renal function. Consequently, no patients required renal replacement therapy and the overall operative mortality was $6 \%$.

Despite advances in critical care, prehospital care, anesthesia care, and postoperative techniques in recent decades, mortality after open repair of rAAA remains remarkably high [2]-[5]. EVAR has been advocated as a less invasive alternative for treatment of non-ruptured and ruptured AAAs [2]-[4] [7]. Its use avoids general anesthesia, secures proximal control with use of an aortic occlusion balloon, reduces blood loss, and circumvents the need for laparotomy in critically ill elderly patients [11]. Although randomized trials demonstrate equivalent in-hospital mortality with EVAR and open repair, large-scale, nationwide, observational studies and meta-analyses have shown EVAR to confer improved in-hospital mortality and morbidity in patients with favorable aneurysm morphology stable enough to undergo imaging [12]. However, it is reported that up to 50\% of rAAA patients have anatomy unsuitable for EVAR because they have a short aortic neck length $(<10$ mm), large aortic neck diameter $(>32 \mathrm{~mm})$, steep aortic neck angulation $\left(>60^{\circ}\right)$, or iliac artery stenosis $(<6 \mathrm{~mm})$. Inadequate neck length, in particular, was most commonly reason for preclusion of EVAR (in 73\% of unsuitable patients) [13]. In such cases, pararenal segments or SC aortic clamping are most likely required.

It is very important to stabilize the hemodynamic status of patients and to prevent fatal complications by prompt bleeding control with proximal aortic clamping and subsequent aortic repair for rAAA. Particularly for rAAA with a short aortic neck length or juxtarenal rAAA, SC and thoracic aortic clamping are methods that may be used to control bleeding in an expeditious manner [2] [11] [14]-[16]. There are several conceivable advantages to SC aortic clamping for repair of rAAA: 1) There is a lesser extent of atherosclerotic disease in the SC aorta compared to that of the IR and pararenal segments; 2) SC aortic clamping is performed at a location distant from the aortic disease process; thus, SC clamping avoids second rupture, clamp encroachment on, or distortion of, the proximal aortic anastomotic site; 3) SC exposure can be easily and expeditiously accomplished through a transperitoneal approach; and 4) extensive mobilization or division of the left renal vein is not necessary to obtain suprarenal control [15] [17]. Considering these advantages, SC aortic clamping has also been applied to non-ruptured pararenal AAA [14]-[17]. However, SC aortic clamping causes a transitory ischemia of the abdominal visceral organs, which can cause fatal ischemic complications.

Postoperative renal dysfunction is the most common complication of aneurysm repair requiring SC aortic clamping [14] [16] [17], and is associated with not only increased in-hospital morbidity and mortality but also adverse long-term outcomes [18] [19]. The effects of renal ischemic time and clamping level on postoperative renal function have been primarily examined in non-ruptured pararenal AAAs. With regard to the effect of renal ischemic time on postoperative renal function, Kudo et al. showed a positive linear relation between ischemic time and transient postoperative renal damage [20], and Dubois et al. showed a linear relation between ischemic time and the likelihood of postoperative renal dysfunction [21]. With regard to the effect of clamping level on postoperative renal function, Sarac et al. showed that SC aortic clamping is associated with postoperative renal dysfunction to a greater extent than is suprarenal clamping, regardless of renal ischemic time [22]. Myers et al. showed that nitric oxide is important in maintaining renal cortical and medullary blood flow and function; in addition to the renal ischemic-reperfusion injury caused by renal blood flow interruption, superior mesenteric artery blood flow interruption induces mesenteric ischemia-reperfusion, which further results in the generation of oxygen-derived free radicals that contribute to decreased renal cortical and medullary nitric oxide synthesis [23]. They also suggested that maintaining visceral blood flow might be helpful in developing strategies to minimize renal ischemia-reperfusion injury [23]. However, if the renal ischemic time does not exceed 20 - 30 minutes, postoperative renal dysfunction does not usually occur, regardless of the clamping level [14] [15] [17]. Similarly, postoperative ischemic visceral injury is rare if the visceral ischemic time does not exceed 20 - 30 minutes [15] [17]. However, for patients undergoing concomitant renal or visceral revascularization for significant atheros- 
clerotic occlusive diseases, operative morbidity, mortality, and complication rates may be high because of preoperative organ failure, complexity of adjunctive renal or visceral revascularization, and prolonged renal/visceral ischemia [17]. In the present study, the SC aortic clamping time was $30 \pm 7$ minutes, and no renal artery revascularization was required, except for one case with para-anastomotic aneurysm rupture. Consequently, SC aortic clamping had no harmful effects on postoperative renal function and was associated with minimal morbidity. Although we have never applied thoracic aortic clamping to rAAA, Büket et al. reported that this technique was associated with a higher mortality rate (62.5\%) than that associated with SC aortic clamping (8.3\%) [15].

The limitations of this study include its retrospective design and the small number of patients. In addition, because of patient selection bias, we cannot simply compare our results to those of previous reports; although we did not perform surgery on patients who had cardiac arrest, in some previous studies surgery was performed for such patients, and these patients were included in the analysis of overall mortality. However, in the present study, patients with preoperative shock also underwent surgery and a successful outcome was achieved.

\section{Conclusion}

We performed SC aortic clamping for rAAA in patients with a short aortic neck length. SC aortic clamping was associated with no harmful effect on postoperative renal function and with minimal mortality and morbidity compared with IR aortic clamping. Forthcoming technological advancements may make it possible to adapt EVAR for patients with unfavorable aneurysm morphology. However, until then, open surgery should be the procedure of choice for patients with unfavorable aneurysm morphology, and SC clamping is effective and safe if the visceral ischemic time is limited to 20 - 30 minutes.

\section{Acknowledgements}

We would like to thank Editage (www.editage.jp) for English language editing.

\section{References}

[1] Colin, M.B. and Elliot, L.C. (2012) Open Repair of Abdominal Aortic Aneurysms. In: Enrico, A., Frank, J.V. and Peter, G., Eds., Haimovici's Vascular Surgery, 6th Edition, Wiley-Blackwell, Oxford, 533-553.

[2] Anain, P.M., Anain Sr, J.M., Tiso, M., Nader, N.D. and Dosluoglu, H.H. (2007) Early and Mid-Term Results of Ruptured Abdominal Aortic Aneurysms in the Endovascular Era in a Community Hospital. Journal of Vascular Surgery, 46, 898-905. http://dx.doi.org/10.1016/j.jvs.2007.06.037

[3] Mureebe, L., Egorova, N., Giacovelli, J.K., Gelijns, A., Kent, K.C. and McKinsey, J.F. (2008) National Trends in the Repair of Ruptured Abdominal Aortic Aneurysms. Journal of Vascular Surgery, 48, 1101-1107. http://dx.doi.org/10.1016/j.jvs.2008.06.031

[4] Starnes, B.W., Quiroga, E., Hutter, C., Tran, N.T., Hatsukami, T., Meissner, M., et al. (2010) Management of Ruptured Abdominal Aortic Aneurysm in the Endovascular Era. Journal of Vascular Surgery, 51, 9-18. http://dx.doi.org/10.1016/j.jvs.2009.08.038

[5] Kunishige, H., Ishibashi, Y., Kawasaki, M., Morimoto, K. and Inoue, N. (2013) Risk Factors Affecting Survival after Surgical Repair of Ruptured Abdominal Aortic Aneurysm. Annals of Vascular Diseases, 6, 631-636. http://dx.doi.org/10.3400/avd.oa.13-00035

[6] Ito, H., Shimono, T., Shimpo, H., Kato, N. and Takeda, K. (2013) Open Surgery for Abdominal Aortic Aneurysm in the Era of endovascular Repair: Comparison with Long Term Results of Endovascular Repair Using Zenith Stentgraft. Annals of Vascular Diseases, 6, 189-194. http://dx.doi.org/10.3400/avd.oa.12-00101

[7] Chang, R.W., Goodney, P., Tucker, L.Y., Okuhn, S., Hua, H., Rhoades, A., et al. (2013) Ten-Year Results of Endovascular Abdominal Aortic Aneurysm Repair from a Large Multicenter Registry. Journal of Vascular Surgery, 58, 324-332. http://dx.doi.org/10.1016/j.jvs.2013.01.051

[8] Rutherford, R.B. (1992) Classification of Ruptured Aortic Aneurysms Aids Comparison of Results. VascSurg Outlook, 4, 1-2.

[9] Fitzgerald, J.F., Stillman, R.M. and Powers, J.C. (1978) A Suggested Classification and Reappraisal of Mortality Statistics for Ruptured Atherosclerotic Infrarenal Aortic Aneurysms. SurgGynecolObstet, 146, 344-346.

[10] Lindsay, T.F. (2010) Abdominal Aortic Aneurysms: Ruptured. In: Cronenwett, J.L. and Johnston, K.W., Eds., Rutherford's Vascular Surgery, 7th Edition, Saunders Elsevier, Philadelphia, 1994-2013.

http://dx.doi.org/10.1016/B978-1-4160-5223-4.00130-X 
[11] Livesay, J.J. and Talledo, O.G. (2013) Endovascular Aneurysm Repair Is Not the Treatment of Choice in Most Patients with Ruptured Abdominal Aortic Aneurysm. Texas Heart Institute Journal, 40, 556-559.

[12] Antoniou, G.A., Ahmed, N., Georgiadis, G.S. and Torella, F. (2015) Is Endovascular Repair of Ruptured Abdominal Aortic Aneurysms Associated with Improved In-Hospital Mortality Compared with Surgical Repair? Interactive Cardiovascular and Thoracic Surgery, 20, 135-139. http://dx.doi.org/10.1093/icvts/ivu329

[13] Slater, B.J., Harris, E.J. and Lee, J.T. (2008) Anatomic Suitability of Ruptured Abdominal Aortic Aneurysms for Endovascular Repair. Annals of Vascular Surgery, 22, 716-722. http://dx.doi.org/10.1016/j.avsg.2008.06.001

[14] Crawford, E.S., Beckett, W.C. and Greer, M.S. (1986) Juxtarenal Infrarenal Abdominal Aortic Aneurysm. Special Diagnostic and Therapeutic Considerations. Annals of Surgery, 203, 661-670. http://dx.doi.org/10.1097/00000658-198606000-00011

[15] Büket, S., Atay, Y., Islamoğlu, F., Yağdi, T., Posacioğlu, H., Alat, I., et al. (1999) Proximal Clamping Levels in Abdominal Aortic Aneurysm Surgery. Texas Heart Institute Journal, 26, 264-268.

[16] El-Sabrout, R.A. and Reul, G.J. (2001) Suprarenal or Supraceliac Aortic Clamping during Repair of Infrarenal Abdominal Aortic Aneurysms. Texas Heart Institute Journal, 28, 254-264.

[17] Nypaver, T.J., Shepard, A.D., Reddy, D.J., Elliott Jr., J.P. and Ernst, C.B. (1993) Supraceliac Aortic Cross-Clamping: Determinants of Outcome in Elective Abdominal Aortic Reconstruction. Journal of Vascular Surgery, 17, 868-876. http://dx.doi.org/10.1016/0741-5214(93)90036-L

[18] Barratt, J., Parajasingam, R., Sayers, R.D. and Feehally, J. (2000) Outcome of Acute Renal Failure Following Surgical Repair of Ruptured Abdominal Aortic Aneurysms. European Journal of Vascular and Endovascular Surgery, 20, 163-168. http://dx.doi.org/10.1053/ejvs.2000.1078

[19] Kopolovic, I., Simmonds, K., Duggan, S., Ewanchuk, M., Stollery, D.E. and Bagshaw, S.M. (2013) Risk Factors and Outcomes Associated with Acute Kidney Injury Following Ruptured Abdominal Aortic Aneurysm. BMC Nephrology, 14, 99. http://dx.doi.org/10.1186/1471-2369-14-99

[20] Kudo, F.A., Nishibe, T., Miyazaki, K., Murashita, T., Yasuda, K., Ando, M., et al. (2004) Postoperative Renal Function after Elective Abdominal Aortic Aneurysm Repair Requiring Suprarenal Aortic Cross-Clamping. Surgery Today, 34, 1010-1013. http://dx.doi.org/10.1007/s00595-004-2871-9

[21] Dubois, L., Durant, C., Harrington, D.M., Forbes, T.L., Derose, G. and Harris, J.R. (2013) Technical Factors Are Strongest Predictors of Postoperative Renal Dysfunction after Open Transperitoneal Juxtarenal Abdominal Aortic Aneurysm Repair. Journal of Vascular Surgery, 57, 648-654. http://dx.doi.org/10.1016/j.jvs.2012.09.043

[22] Sarac, T.P., Clair, D.G., Hertzer, N.R., Greenberg, R.K., Krajewski, L.P., O’Hara, P.J., et al. (2002) Contemporary Results of Juxtarenal Aneurysm Repair. Journal of Vascular Surgery, 36, 1104-1111. http://dx.doi.org/10.1067/mva.2002.129638

[23] Myers, S.I., Wang, L. and Myers, D.J. (2007) Loss of Renal Function and Microvascular Blood Flow after Suprarenal Aortic Clamping and Reperfusion (SPACR) above the Superior Mesenteric Artery Is Greatly Augmented Compared with SPACR above the Renal Arteries. Journal of Vascular Surgery, 45, 357-366.

http://dx.doi.org/10.1016/j.jvs.2006.10.045 


\section{Abbreviations}

AAA: Abdominal Aortic Aneurysm;

rAAA: ruptured AAA;

EVAR: Endovascular Aneurysm Repair;

SC: Supraceliac;

IR: Infrarenal;

CT: Computed Tomography;

ACS: Abdominal Compartment Syndrome;

TAC: Temporary Abdominal Closure;

BUN: Serum Blood Urea Nitrogen;

CR: Serum Creatinine;

eGFR: estimated Glomerular Filtration Rate. 